\title{
A DSP Architecture for Cochlear Implants
}

\author{
Eric D. Marsman, \\ Robert M. Senger \\ University of Michigan \\ Ann Arbor, MI 48109-2122, USA \\ \{emarsman, rsenger\}@eecs.umich.edu
}

\author{
Gordon A. Carichner, Sundus Kubba, \\ Michael S. McCorquodale \\ Mobius Microsystems, Inc. \\ Detroit, MI 48226-1686, USA \\ \{carichner, kubba, mccorquodale\}@mobiusmicro.com
}

\author{
Richard B. Brown \\ University of Utah \\ Salt Lake City, UT 84112-1109, USA \\ brown@coe.utah.edu
}

\begin{abstract}
This paper describes a low-power DSP architecture for use in cochlear implants. The microsystem, fabricated in TSMC $0.18 \mu \mathrm{m}$ CMOS, consumes $1.79 \mathrm{~mW}$ from a $1.2 \mathrm{~V}$ supply and occupies an area of $9.18 \mathrm{~mm}^{2}$ while providing the necessary programmability for high speech comprehension by patients. Standby power consumption is $330 \mu \mathrm{W}$.
\end{abstract}

\section{INTRODUCTION}

The primary design considerations for battery powered bio-medical implant systems are safety, reliability, area, and power consumption. The work discussed here focuses on reducing the area and power consumption of cochlear implants (CIs) by integrating the signal processing capability required into an SoC. Increased integration of components into a single SoC will enable fully-implantable CIs in the near future; something that is not possible with traditional CI approaches.

The microsystem described here combines an energyefficient microcontroller unit (MCU), a low-power DSP core, and a monolithic self-referenced hybrid temperaturecompensated LC oscillator (TC-LCO) and ring oscillator clock reference into a single bulk CMOS SoC. This work is being developed as part of the Wireless Integrated MicroSystems (WIMS) Engineering Research Center (ERC) [1].

The next section gives a brief description of CIs, followed by the architecture of the microsystem, testing results, and comparisons to other CI approaches.

\section{COCHLEAR IMPLANTS}

CIs are medical devices implanted in patients who have a degeneration or absence of sensory hair cells in the inner ear. In a properly functioning ear, frequency selective hair cells in the cochlea generate electrical signals that are detected by

This work was supported primarily by the Engineering Research Centers Program of the National Science Foundation under award number EEC9986866. neurons and transmitted via the auditory nerve to the brain for processing. The purpose of a CI is to bypass non-functional hair cells by directly stimulating the cochlea with current pulses. To date, over 90,000 people worldwide have received cochlear implants [2].

The major components of a CI include a microphone, signal processor, implant electrodes, and batteries. The microphone, fitted into or placed behind the patient's ear, captures the acoustic signal and converts it to an electrical signal. Using this information, the signal processor calculates the type and level of stimulation to deliver through the electrodes, which are surgically inserted into the cochlea. Large variations in signal processing algorithms exist due to diverse implementation methods and differing parameters among patients.

Typical commercial CIs use an off-the-shelf, softwareprogrammable DSP to perform the signal processing. In this paper we report the development of a fully integrated, lowpower, DSP core to perform the Continuous Interleaved Sampling (CIS) algorithm. CIS is by far the most popular processing algorithm among patients and manufacturers. Research studies have demonstrated that it provides the highest speech comprehension rates among patients [3]. As shown in Fig. 1, a high-pass filter (HPF) attenuates vowel sounds before the band-pass filters (BPFs) split the signal into $n$ channels ( $n=16$ for this design). Following envelope detection by the low-pass filter (LPF) and rectifier, nonlinear dynamic range compression maps the electrical representation of acoustic signals to a representation of current levels. Lastly, non-overlapping bi-phasic pulses are generated and delivered through the stimulation electrodes as current pulses to the cochlea.

Certain parameters of the signal processing algorithm must be variable in order to provide optimal hearing for the patient [4]. The most important of these are the filter cutoff frequencies, compression function, number of channels, 


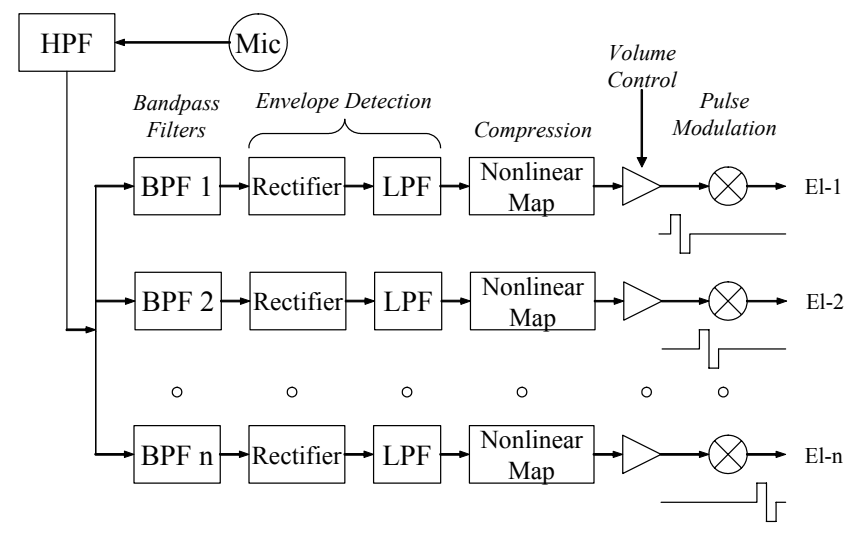

Figure 1. CIS signal processing algorithm

channel to electrode assignment, pulse duration, and pulse rate. Our CI microsystem allows individual patients to be custom fitted by programming each of these parameters via the MCU.

Reference [5] is the lowest reported power consumption for an analog signal processor for CIs. This 16-channel CIS processor consumes $251 \mu \mathrm{W}$ from $2.8 \mathrm{~V}$ and occupies $88.9 \mathrm{~mm}^{2}$ in an AMI $1.5 \mu \mathrm{m}$ BiCMOS process. This is 7.1 times less power, but nearly an order of magnitude more area than the presented microsystem. While this is an impressive power consumption achievement, the analog system sacrifices programmability and flexibility, especially when it comes to stimulation profiles and dynamic range compression. These two signal processing factors are very important in determining patient speech comprehension.

For software programmable DSPs, 35 million operations per second is an aggressive estimate of the processing capability required to perform a 16-channel CIS implementation that includes the signal processing and communication with the $\mathrm{ADC}$ and electrode array. This typically requires $45 \mathrm{~mW}$ to $90 \mathrm{~mW}$ of power, depending on the algorithm implementation and processor selection [6][7]. Software programmable DSP approaches consume too much power and area to be part of a fully-implanted CI system.

\section{DSP MicRosystem ARCHITECTURE}

Fig. 2 shows the complete microsystem architecture consisting of the MCU, DSP, and hybrid clock source. An overview of a prior version of the MCU core has been given in [8]. Improvements upon the previous MCU for this work include a more efficient ISA to relieve compiler bottlenecks, additional communication interfaces, and decreased power consumption. The 16-bit MCU contains a 3-stage pipeline, $32 \mathrm{kB}$ of on-chip memory, a loop cache, and several peripheral communication interfaces. This makes the microsystem well suited for other bio-medical or environmental monitoring applications.

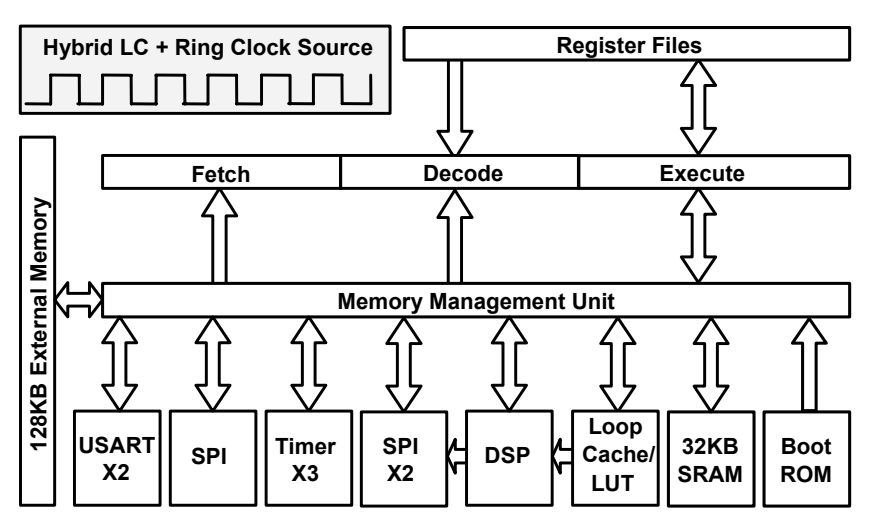

Figure 2. Microsystem architecture

The DSP and MCU share control over two SPI interfaces and the loop cache/look-up-table (LUT). The SPI interfaces are intended to communicate with an external ADC and the implanted electrodes. Control registers in the DSP, written by the MCU software, are read by the MCU memory management unit to give control of the read and write data buses for these components to either the DSP or MCU. These control bits also determine the clock domain in which the peripheral ports and the loop cache operate. The microsystem software can select different operating frequencies for the MCU and DSP separately to provide maximum operating point flexibility [9].

The self-referenced hybrid clock source consists of a highly-accurate TC-LCO and an independent tunable ring oscillator. No external crystal or PLL is required. The synthesizer supports a reduced-power standby mode in which the TC-LCO is powered down while the system operates from the low power, low frequency ring oscillator. The entire clock synthesizer occupies $0.25 \mathrm{~mm}^{2}$ of silicon area.

Fig. 3 is a block diagram of the fully-synthesizable signed-magnitude fixed-point DSP core. The HPF, BPFs, and LPFs are implemented as cascaded infinite impulse response (IIR) stages due to the low memory requirements and simplicity of the hardware. They are $1^{\text {st }}, 6^{\text {th }}$, and $4^{\text {th }}$ order, respectively. All filter coefficients are programmable by the MCU to provide the required flexibility for patient fitting procedures. All filters obtain a vast reduction in area, due to the multiply-intensive nature of filters, by sharing the same cascade stage hardware. Storage for the filter coefficients and output data from each datapath stage is a significant requirement on the architecture. Clock gating is performed on these registers to reduce the power consumed by the clock tree.

Compressing the dynamic range, and therefore the number of bits required to encode the signal amplitude, saves power and area by reducing the datapath width from sixteen to eight bits for all downstream calculations and storage. 
This logarithmic compression is done using the low-power loop cache to store the LUT data. By allowing the MCU software to control the data in the 512-entry LUT, the patient can have the compression function individually optimized.

Pulse rate, channel to electrode assignment, and pulse duration are all programmable in the modulator via the MCU interface. The current implementation allows for any bi-phasic pulse stimulation information to be sent to the electrodes through one of the system's SPI interfaces. The maximum pulse rate is 3000 pulses per second per channel. In addition, if more complex stimulation profiles are desired, the MCU can read the data coming out of the DSP volume stage and perform computations to calculate the appropriate pulse characteristics. It can then take control of the SPI interface and send information to the implanted electrodes, bypassing the DSP modulator finite state machine (FSM). This makes the microsystem a useful tool for investigating experimental stimulation profiles.

The parallel nature of the CIS algorithm provides for a significant reduction in hardware by pipelining the datapath and allowing all channels to share the same hardware for filters, LUT, volume control, and pulse modulation. Control circuitry is also simplified by allowing the FSMs in the control unit to reuse states for each channel.

The DSP core has four operating modes: stimulation, programming, test, and sleep. For typical operation, the DSP will be in stimulation mode and will process samples and generate stimulation pulses automatically. The MCU can be in standby mode during this time. Input data is received from the external ADC through one of the shared SPI interfaces.

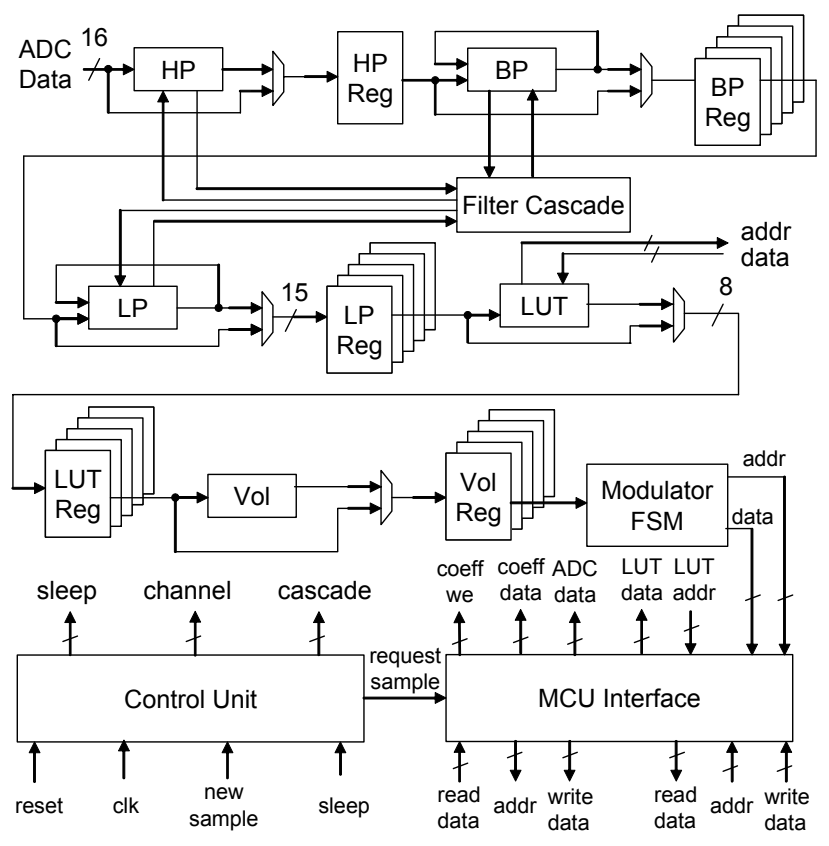

Figure 3. CIS DSP architecture block diagram
Programming mode will allow the MCU to set up all filter coefficients, LUT data, and the stimulation profile. Test mode allows for any of the datapath stages to be bypassed via the multiplexors at each output node to provide observability and controllability over each component in the DSP. Sleep mode allows all DSP components to be shut down in order to conserve power. While in stimulation mode, any unused datapath stages are shut down through the control unit by utilizing the existing sleep mode circuitry.

Assuming a $24 \mathrm{kHz}$ front-end ADC, which is standard for speech processing within the human audible range of 0 to $10 \mathrm{kHz}$, the DSP must operate at $3 \mathrm{MHz}$ to provide adequate output data for high pulse rate stimulation. This calculation is based on the DSP processing time of a single sample and the data transmission rate to the electrodes.

\section{MEASURED RESUlts}

Fig. 4 shows the fabricated microsystem in TSMC $0.18 \mu \mathrm{m}$ mixed-mode bulk CMOS containing 2.3 million transistors and occupying $9.18 \mathrm{~mm}^{2}$. Table 1 shows the measured data, averaged across ten devices, taken on an HP82000 D200/D400 digital tester. $1.79 \mathrm{~mW}$ is the lowest reported active power consumption for a CI specific DSP.

Fig. 5 shows the capability of the DSP to scale frequency as well as supply voltage. Operating with fewer than sixteen active channels will allow the DSP to reduce its operating frequency and power consumption. When improving electrode technology allows more electrodes to be inserted into

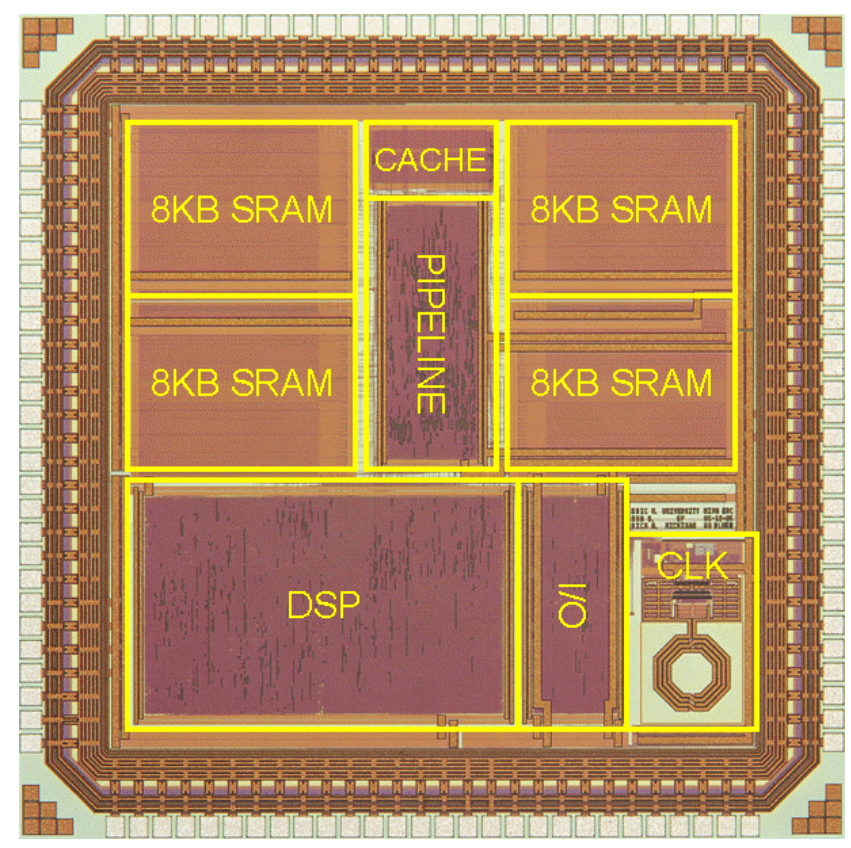

Figure 4. Die micrograph of fabricated microsystem 
Table 1. Measured Power Consumption Data.

\begin{tabular}{|c|c|c|c|c|}
\hline \multirow{2}{*}{$\begin{array}{c}\text { Simulation } \\
\text { Conditions }\end{array}$} & $\begin{array}{c}|c| \\
\text { DSP } \\
\text { Mode }{ }^{a}\end{array}$ & Standby & $\begin{array}{c}\text { VSP } \\
\text { Mode }{ }^{a}\end{array}$ & Standby \\
\cline { 2 - 5 } & 1630 & 170 & 440 & 60 \\
\hline Core $(\mu \mathbf{W})$ & 120 & 120 & 30 & 30 \\
\hline Mem $(\mu \mathbf{W})$ & 2460 & 270 & 1140 & 60 \\
\hline DSP $(\mu \mathbf{W})$ & 760 & 760 & 180 & 180 \\
\hline CLK $(\mu \mathbf{W})$ & 4970 & 1320 & 1790 & 330 \\
\hline Total $(\mu \mathrm{W})$ & & & \\
\hline
\end{tabular}

a.The DSP operating frequency is $3 \mathrm{MHz}$.

the cochlea, requiring more signal processing from the system, increasing the number of channels in this DSP architecture to more than 16 channels requires only the addition of more register storage for the additional filter coefficients and data outputs. No additional control or computational hardware would be required due to the pipelined nature of the architecture. Because this architecture is fully-synthesizable, migration to a more advanced bulk CMOS or SOI process will require minimal work while achieving large reductions in power and area.

\section{CONCLUSiON}

This paper describes an expandable, programmable, fully-synthesizeable, low-power, low-area DSP architecture that performs the CIS algorithm. This microsystem achieves the lowest reported digital CI power consumption of $1.79 \mathrm{~mW}$ in active mode and $330 \mu \mathrm{W}$ in sleep mode; it occupies $9.18 \mathrm{~mm}^{2}$ of Si area. Future improvements to the register storage will reduce area and power consumption. The amount of on-chip memory can also be reduced to decrease the area in a CI-only application implementation.

Future expansion to more than sixteen channels will increase the power and area of analog signal processing systems on a linear scale, while the digital WIMS CI system architecture achieves a superior scaling factor. Migration to a more advanced process will improve the performance of the digital system, but analog systems will not obtain the same benefits [10].

\section{ACKNOWLEDGEMENTS}

Fabrication of this work at TSMC was funded by the MOSIS Educational Program. The authors wish to thank Artisan Components for supplying the digital standard cell libraries and memory generators and Synopsys, Cadence, and Mentor Graphics for providing CAD tools through their university programs. Thank you also to Dr. Blake Wilson

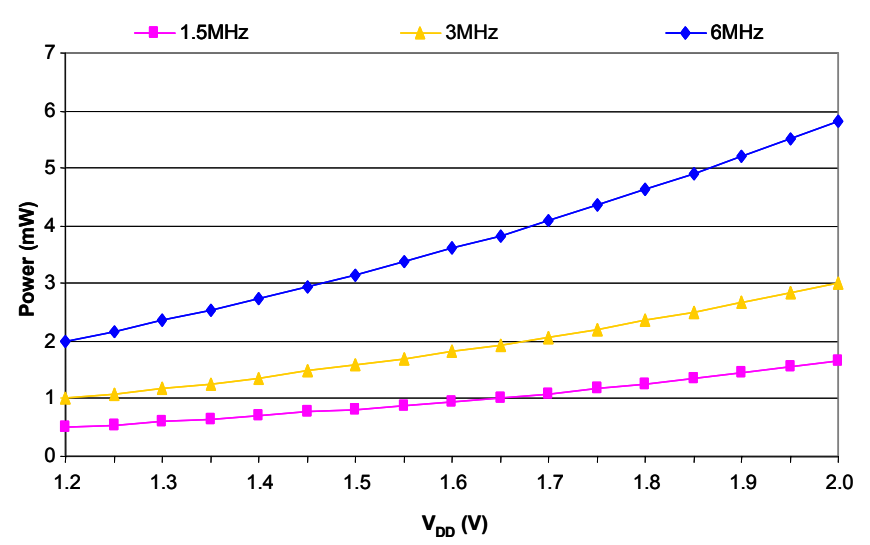

Figure 5. Measured power versus $\mathrm{V}_{\mathrm{DD}}$ scaling at different frequencies

and Reinhold Schatzer at RTI for sharing their CI and CIS expertise.

\section{REFERENCES}

[1] Center for Wireless Integrated Microsystems (WIMS), An NSF Engineering Research Center, [Online]. Available: http://www.wimserc.org/, 2005.

[2] P. Bhatti, S. Lee, and K. Wise, "A 32-site 4-channel cochlear electrode array," Intl. Solid State Circuits Conf. (ISSCC), pp. 50-51, Feb. 2006.

[3] P. Loizou, "Mimicking the human ear," IEEE Signal Process. Mag., vol. 15 , no. 5, pp. 101-130, Sep. 1998.

[4] B. S. Wilson, et al. "Importance of patient and processor variables in determining outcomes with cochlear implants," J Speech Hear Res., vol. 36, pp. 373-379, 1993.

[5] R. Sarpeshkar, et al., "An analog bionic ear processor with zero-crossing detection," Intl. Solid State Circuits Conf. (ISSCC), pp. 78-79, Feb. 2005.

[6] 0. Poroy and P. Loizou, "Development of a speech processor for laboratory experiments with cochlear implant patients," Intl. Conf. on Acoustics, Speech, and Signal Processing (ICASSP), pp. 3626-3629, June, 2000 .

[7] Texas Instruments, "TMS320C5402 fixed-point digital signal processor reference manual," 2000.

[8] E. Marsman, et al., "A 16-bit low-power microcontroller with monolithic MEMS-LC clocking," Intl. Symp. on Circuits and Systems (ISCAS), May 2005, pp. 624-627.

[9] R. Senger, et al., "Low-latency, HDL-synthesizable dynamic clock frequency controller with hybrid $L C$ clocking," Intl. Symp. on Circuits and Systems (ISCAS), May 2006.

[10] A. J. Annema, "Analog circuit performance and process scaling," Transactions on Circuits and Systems II: Analog and Digital Signal Processing, vol. 46, pp. 711-725, June, 1999. 